\title{
Chemisches Recycling kunststoffhaltiger Abfälle - Das Potenzial der Pyrolyse
}

\author{
Dieter Stapf, Hans Leibold, Niklas Netsch, Frank Richter, Savina P. Yogish und \\ Michael Zeller, Karlsruher Institut für Technologie (KIT), Institut für Technische \\ Chemie, Postfach 3640, 76021 Karlsruhe, dieter.stapf@kit.edu
}

Chemisches Recycling (Solvolyse, Pyrolyse, Vergasung) von Kunststoffabfällen stellt in Ergänzung zu den bestehenden Sammel- und mechanischen Sortiersystemen mit derzeit begrenzter Recyclingquote und Produktqualität eine wesentliche Option dar, um den Kohlenstoffkreislauf zu schließen. Hierbei werden Sekundärrohstoffe der Chemie zur Herstellung von Neuware erzeugt, als Beitrag zu den geforderten Recyclingquoten für Kunststoffe und zur klimaneutralen chemischen Produktion.

Die in Frage kommenden realen Abfallströme sind in der Regel Mischungen von Kunststoffen mit mineralischen Bestandteilen und anderem organischen Material, die physikalisch nicht vollständig trennbar sind. Zur Auswahl stehende chemische Technologien wurden im Fall der Pyrolyse, die derzeit im industriellen Fokus steht, bisher nur für ausgewählte Abfälle wie z.B. thermoplastische Verpackungsmaterialien eingesetzt, meist in kleinerem Maßstab. Die erforderlichen Produktqualitäten werden derzeit großtechnisch mit realen Abfällen kaum erreicht. Im Gegensatz zu Vergasungsprozessen ist die Produktzusammensetzung der Pyrolyse nicht thermodynamisch kontrolliert. Insbesondere für Duroplasten oder technische Kunststoffe liegen auch kaum nachvollziehbar ermittelte Produktverteilungen in Abhängigkeit von den Pyrolysebedingungen vor. Die technologische Reife der Optionen an thermochemischen Recyclingverfahren ist zur unmittelbaren Umsetzung mit relevanten Mengenströmen nicht hoch genug /1/.

Voraussetzung zur Beurteilung von Pyrolyseprozessen für gemischte Abfälle ist die Kenntnis der Produktausbeuten (Permanentgase, kondensierbare Fraktionen, Koks/Asche). Hierzu wurden exemplarische Kunststoffmischabfälle einheitlich aufbereitet, im technisch relevanten Maßstab pyrolysiert und Prozess sowie Produktverteilungen energetisch und stofflich bilanziert. Beispiele sind Sortierreste aus der Leichtverpackungssammlung, Kunststoffreste der Gewerbeabfallaufbereitung, Großschredder- und Kleinschredder-Abfälle (Automobil bzw. Elektro- und Elektronik), oder Kunststoffabfälle aus dem Baubereich (Modell-Wärmedämmverbundsysteme),

Die Ergebnisse werden auf großtechnische Übertragbarkeit ausgewertet und anhand von Indikatoren systematisch gegenübergestellt. Technologische sowie ökologische und ökonomische Fragestellungen werden diskutiert.

11/ „Thermal Processes for Feedstock Recycling of Plastics Waste“, www.bkvgmbh.de/infothek/studien.html, 2019 